\title{
The Formation of CIRs at Stream-Stream Interfaces and Resultant Geomagnetic Activity
}

I. G. Richardson

NASA Goddard Space Flight Center, Greenbelt, Maryland and the Department of Astronomy, University of Maryland, College Park

\begin{abstract}
Corotating interaction regions (CIRs) are regions of compressed plasma formed at the leading edges of corotating high-speed solar wind streams originating in coronal holes as they interact with the preceding slow solar wind. Although particularly prominent features of the solar wind during the declining and minimum phases of the 11-year solar cycle, they may also be present at times of higher solar activity. We describe how CIRs are formed, and their geomagnetic effects, which principally result from brief southward interplanetary magnetic field excursions associated with Alfvén waves. Seasonal and long-term variations in these effects are briefly discussed.
\end{abstract}




\section{The formation of corotating interaction regions}

The first in-situ observations of the solar wind were returned by the Mariner 2 spacecraft en route to Venus in 1962 . They revealed that the solar wind at that time, during the latedeclining phase of solar cycle 19, was organized into streams of fast $(>>400 \mathrm{~km} / \mathrm{s}$ ) flows, separated by intervals of slower solar wind. The fast flows lasted for several days, and tended to recur at the solar rotation interval (sidereal period $=25.38$ days, or 27.28 days as viewed from Earth), suggesting they were long-lived, spatial features corotating with the Sun [Neugebauer and Snyder, 1966]. Similar high-speed streams have subsequently been observed in the inner heliosphere, for example by the Helios 1 and 2 spacecraft at 0.3 - 1 AU [e.g., Schwenn, 1990], in the outer heliosphere by Pioneers 10 and 11 and Voyagers 1 and 2 [e.g., Smith and Wolfe, 1979; Burlaga et al., 1983; Gazis and Lazarus, 1983; Gazis, 1984], as well as by Ulysses [e.g., McComas et al., 2000] and numerous near-Earth spacecraft.

Corotating interaction regions (CIRs) [Belcher and Davis, 1971; Smith and Wolfe, 1979] form at the leading edges of corotating high-speed streams as they collide with the preceding slower solar wind. As predicted by the Parker [1958] solar wind theory, solar wind flow streamlines, and the magnetic field lines that are carried out with the solar wind plasma, are twisted into Archimedian spirals of the form

$$
r-r_{o}=-V\left(\phi-\phi_{o}\right) /(\Omega \cos \theta),
$$

where $r$ is the heliocentric distance, $V$ is the solar wind speed, $\Omega$ is the solar angular velocity, $\theta$ and $\phi$ arc the heliolatitude and heliolongitude of the observer, and $r_{o}$ and $\phi_{o}$ are the heliocentric distance and heliolongitude of the initial plasma position at the Sun. At low latitudes, streamlines are inclined at an angle

$$
\psi=\arctan (r \Omega / V)
$$

to the outward radial direction. Thus, streamlines in faster solar wind follow spirals that are less tightly wound. The resulting collision of the leading edge of a fast solar wind stream with the slower solar wind ahead of it forms a region of enhanced pressure, the corotating interaction region, that lies approximately along the Archimedian spiral [Parker, 1963]. At $1 \mathrm{AU}, r \Omega \approx 400$ $\mathrm{km} / \mathrm{s} \sim V$, so flow streamlines, the interplanetary magnetic field, and CIRs are typically inclined at $\psi \sim 45^{\circ}$ to the radial direction.

Figure 1 [Richardson et al., 1996, after Belcher and Davis, 1971] shows the formation of CIRs at the leading edges of two high-speed streams, as viewed from above the north solar pole. Dotted lines indicate representative magnetic field lines/streamlines in the slow and fast solar wind. Variations in plasma parameters at $\sim 1$ AU associated with the stream interactions are also shown. It is convenient to identify four regions: the ambient, undisturbed, slow solar wind (S); the compressed, accelerated, originally slow solar wind plasma ( $\left.\mathrm{S}^{\prime}\right)$; the compressed, decelerated, fast-stream plasma ( $\left.F^{\prime}\right)$, and the ambient, undisturbed, fast-stream plasma (F). The $\mathrm{S}^{\prime}$ and $\mathrm{F}^{\prime}$ regions form the interaction region, characterized by enhanced plasma densities and magnetic field intensities.

An important feature within a CIR is the boundary between the $S^{\prime}$ and $F^{\prime}$ regions, the "stream interface" [Burlaga, 1974; Gosling et al., 1978; Schwenn, 1990; Forsyth and Marsch, 1999; Crooker et al., 1999]. This is typically characterized by a relatively abrupt fall in plasma density $(n)$, and increases in the plasma proton temperature $\left(T_{p}\right)$, solar wind speed, and specific entropy $\propto T_{p} / n^{\gamma-1}$, where $\gamma$ is the ratio of specific heats [Intriligator and Siscoe, 1994]. The stream-stream interaction tends to deflect solar wind to the west (i.e., in the sense of rotation) ahead of the interface and to the east following the interface. Belcher and Davis [1971] suggested that the interface originates as a sharp transition between the two flows near the Sun, ideally a tangential discontinuity. Though chal- 
lenged by Burlaga [1974], who proposed instead that a gradual transition near the Sun becomes steepened by the stream-stream interaction, this interpretation is now generally accepted following the work of Gosling et al. [1978]. Thus, for example, the decrease in density across the interface arises because slow solar wind ahead of the interface is intrinsically denser than the fast solar wind. Changes in solar wind composition across the interface [e.g., Wimmer-Schweingruber et al., 1997] are also consistent with the interface separating slow and fast solar wind. Only 30\% of streams at $1 \mathrm{AU}$ include a clear, discontinuous interface, the fraction increasing to $\sim 50 \%$ if slightly broader structures are also considered [Gosling et al., 1978; Schwenn, 1990]. In some cases, CIRs may include multiple interface-like structures that may be multiple crossings of a wavy boundary separating slow and fast solar wind [Wimmer-Schweingruber et al., 1997].

A close relationship between the stream structure and the recurring sunward- or anti-sunwarddirected "sector" structure of the interplanetary magnetic field was first noted by Wilcox and Ness [1965]. The sector structure is now known to be associated with crossings of the heliospheric current sheet (HCS), which is embedded in slow, dense solar wind emerging from the "streamer belt" that typically overlays the solar magnetic equator. Thus, the HCS is often crossed in the slow solar wind preceding a CIR, and may be incorporated into the CIR along with the slow solar wind [e.g., Pizzo and Gosling, 1994]. Each high-speed stream then has a specific magnetic field polarity reflecting the direction of the field imposed at the Sun.

By tracing spiral streamlines in high-speed flows back to the solar corona, large regions of weak coronal X-ray emission, often persisting for several solar rotations, have been identified as the source regions of corotating high-speed flows [e.g., Krieger et al., 1973; Zirker, 1977]. Such "coronal holes" are also evident as regions of reduced ultraviolet emission or weak scattered visible light, and are clearly visible in FeXV images from the Extreme Ultraviolet Imaging Telescope (EIT) on the SOHO spacecraft (Figure 2).

Figure 3 shows CIRs at the leading edges of three corotating high-speed streams observed during one 27-day period by the near-Earth Advanced Composition Explorer (ACE) and IMP 8 spacecraft in December 1999 - January 2000, which exhibit the features discussed above. The data illustrated include the magnetic field intensity, polar and azimuthal angles, the plasma proton temperature, density, speed, flow angle, and $\mathrm{O}^{7} / \mathrm{O}^{6}$ and $\mathrm{Mg} / \mathrm{O}$ ratios, all from $\mathrm{ACE}$, and the cosmic ray intensity from IMP 8 (specifically, the count rate of the GME anticoincidence guard [Richardson, 2004]). Dashed vertical lines within the magnetic field intensity and plasma density enhancements associated with the CIRs indicate probable stream interface crossings. These are characterized by decreases in density, increases in solar wind speed and proton temperature, the solar wind flow angle moving through the radial direction, changes in the solar wind composition $\left(\mathrm{O}^{7} / \mathrm{O}^{6}\right.$ and $\mathrm{Mg} / \mathrm{O}$ decrease $)$ and the onset of cosmic ray modulations which extend through the high-speed streams. Heliospheric current sheet crossings (abrupt $\sim 180^{\circ}$ changes in field azimuth) occur within the first CIR a few hours ahead of the interface, close to the interface in the second CIR, and near the leading. edge of the third CIR. Note that these streams were observed near solar maximum - corotating streams were prominent in the near-Earth solar wind during 1998 - early 2001 [e.g., Richardson et al., 2002].

As predicted by Hundhausen [1973a, b], CIRs can lead to the formation of shocks that corotate with the Sun. The enhanced plasma pressure within a CIR

$$
P=n k\left(T_{e}+T_{p}\right)+B^{2} / 2 \mu_{o}
$$

(where $T_{e}$ is the plasma electron temperature and $B$ is the magnetic field intensity) causes the CIR to expand into the ambient solar wind. Since the magnetosonic speed in the solar wind

$$
V_{f}=\sqrt{V_{A}^{2}+V_{s}^{2}}
$$


where $V_{A}=B / \sqrt{\mu_{o} \rho}$ is the Alfvén speed, and $V_{s}=\sqrt{5 P / 3 \rho}$ is the sound speed ( $\rho$ is the plasma mass density), decreases with increasing distance from the Sun, the expanding boundaries of the CIR may eventually steepen into shocks. A "forward" shock propagating into the slow solar wind and away from the Sun may form at the CIR leading edge while a "reverse" shock, propagating into the fast solar wind and toward the Sun, may develop at the trailing edge (see Figure 1). Pioneer 10/11 and Voyager 1/2 observations have demonstrated that CIR shocks tend to form beyond 2 AU [e.g., Gosling et al., 1976; Hundhausen and Gosling, 1976; Smith and Wolfe, 1976, 1977; Gazis and Lazarus, 1983]. However, they may occasionally form by 1 AU [e.g., Burlaga, 1970; Lazarus et al., 1970; Chao et al., 1972; Richardson and Zwickl, 1984; Berdichevsky et al., 2000] or even closer to the Sun [Schwenn, 1990].

Figure 4 [Crooker et al., 1999; Richardson, 2004] illustrates the development of a CIR beyond $1 \mathrm{AU}$. The left-hand panel shows energetic particle, solar wind plasma, and magnetic field observations at IMP 8 (1 AU) of a CIR in April, 1974. The interface (I) and two possible locations of developing reverse shock-like features (R?) are identified. The middle panel shows similar Pioneer 11 observations at $3.8 \mathrm{AU}$. Here, the CIR was bounded by fully developed forward $(F)$ and reverse $(R)$ shocks, while the interface may be identified in the middle of the CIR. Note that the heliospheric current sheet that was located in the slow solar wind ahead of the CIR at $1 \mathrm{AU}$, is now incorporated into the CIR, and crossed in a data gap ahead of the interface. At Pioneer 10 (5.2 AU; right-hand panel), again the forward and reverse shocks and stream interface may be identified, and the current sheet is ahead of the interface. Thomas and Smith [1981] found that the forward shock had overtaken the current sheet in the majority of CIRs beyond 5 AU.

The bottom graphs in Figure 4 show how enhanced magnetic field turbulence at 1 AU (here measured by the sum of the squares of the magnetic field component variances) is confined to the region following the stream interface and extends into the high-speed stream. At Pioneer 11 and then Pioneer 10, however, the turbulence (represented by the power at wavenumbers of $1.6-3.3 \times 10^{-5} \mathrm{~km}^{-1}$; T. Horbury, private communication, 1999) becomes distributed throughout, and is largely confined to, the CIR. The top panels show the intensity of $\sim 4 \mathrm{MeV}$ protons accelerated in the vicinity of CIRs and the modulation of $\geq 60 \mathrm{MeV}$ galactic cosmic rays. See Richardson [2004], and references therein, for further discussion of interplanetary energetic particle effects associated with CIRs.

Although corotating high-speed streams may be present in the near-Earth solar wind at all stages of the solar cycle [e.g., Richardson et al., 2002], nevertheless a profound change in the latitudinal structure of high-speed streams occurs between solar minimum and solar maximum. This was first inferred from interplanetary scintillation measurements [Rickett and Coles, 1991] and later confirmed by in-situ observations made by the Ulysses mission, launched in October 1990 to explore the heliosphere to high heliographic latitudes [e.g., Balogh et al., 2001, and references therein]. At solar minimum, the most prominent coronal holes form around the poles of the Sun. Thus, Ulysses observed persistent high-speed coronal hole flows above $\sim 30^{\circ}$ latitude [McComas et al., 2000]. At lower latitudes, CIRs form by interactions between high-speed streams originating from equatorward extensions of the polar coronal holes (e.g., Figure 2(a)) and slow solar wind from the streamer belt. Consistent with this configuration, CIR forward (reverse) shocks observed by Ulysses were found to be propagating to lower (higher) latitudes in the slow (fast) solar wind [Gosling et al., 1993; Gosling, 1996; Riley et al., 1996].

At solar maximum, the polar coronal holes shrink, and Ulysses observed variable solar wind speeds at all latitudes [McComas et al., 2001, 2003]. High-speed flows near the Earth typically emerge from small, lower latitude, coronal holes [e.g., Bravo et al., 1998; Luhmann et al., 
2002; Neugebauer et al., 2002; Wang and Sheeley, 2003]. Figure 2(b) shows the low-latitude coronal hole that gave rise to the second highspeed stream in Figure 3.

For further information on the formation and characteristics of CIRs, see Schwenn and Marsch [1990], chapters 7 and 8 of Burlaga [1995], Volume 89, Nos. 1 -2 of Space Science Reviews [1999], and Balogh et al. [2001].

\section{Geomagnetic activity associated with CIRs and corotating high-speed streams}

Enhanced geomagnetic activity is a consequence of an increase in the rate of energy transfer from the solar wind into the Earth's magnetosphere. This is largely determined by the strength and orientation of the interplanetary magnetic field, the solar wind speed and density. One formulation (not including density) is the $\epsilon$ function of Perreault and Akasofu [1978], $\epsilon=l_{o}^{2} V B^{2} \sin ^{4}(\theta / 2)$, where $l_{o}^{2}$ is the area of the magnetopause through which the energy enters, and $\theta$ is the "clock angle" of the IMF relative to the Sun-Earth line. Although increased energy transfer is expected in faster solar wind, the typical factor of $\sim 2-3$ variation in solar wind speed is much less than the variation in the magnetic field dependence, which reflects the efficient energy transfer that occurs when the IMF has a southward component, facilitating reconnection between the solar wind and magnetospheric magnetic fields [Dungey, 1961].

The strongest geomagnetic "storms" occur sporadically and are almost invariably produced by interplanetary transients (interplanetary coronal mass ejections, ICMEs) resulting from coronal mass ejections at the Sun [Gosling et al., 1991; Richardson et al., 2001; see also section 3.2]. Tsurutani and Gonzalez [1997] give an overview of the interplanetary conditions that drive geomagnetic storms associated with solar wind transients, including southward fields in the CMErelated material or in the "sheath" of compressed plasma ahead of the transient.

Geomagnetic activity associated with CIRs and high-speed streams has a rather different nature. Most characteristically, it tends to recur at the solar rotation period. The existence of recurring geomagnetic activity was first clearly established by Maunder [1905] who noted that periods of enhanced geomagnetic disturbances during 1882 to 1903 were ordered by solar longitude. He inferred that some "definite and restricted areas" on the Sun gave rise to these geomagnetic disturbances. Furthermore, sunspots did not have to be present, and he could find no better relationship with faculae or prominences. Maunder concluded that the recurrence could only be explained "by supposing that the Earth has encountered, time after time, a definite stream ... which, continually supplied from one and the same area of the Sun's surface, appears to us, at our distance, to be rotating with the same speed as the area from which it arises". A stream diameter of $\sim 20^{\circ}$ of solar longitude was suggested by the typical duration of the disturbances. This removed the criticism by Lord Kelvin (among others), on the grounds of the unreasonable energy required, of the then-prevailing concept that geomagnetic activity was caused by "magnetic waves" expanding away from the Sun equally in all directions.

Further insight into the origin of recurrent geomagnetic activity came from the work of Greaves and Newton [1929], who demonstrated (see Figure 1 of Cliver [1995]) that recurrence is a property of smaller geomagnetic storms, and is not exhibited by major storms even though these are often associated with large sunspot regions which may persist for more than one solar rotation. Bartels [1932] extended this work using stacked 27-day plots of the geomagnetic C9 index for 1906 to 1931. A plot of C9 for 1971 1974, during the decline of cycle 20, is shown in Figure 5. Numbers of different "weights" help to visualize the variations in the daily $\mathrm{C} 9$ index and sunspot number (R9). At high solar activity levels, geomagnetic activity is dominated by spo- 
radic, often intense storms associated with transients. As the sunspot number declines, moderate, recurrent geomagnetic activity becomes predominant, as Bartels noted (see also Bartels [1940]). He argued that sunspots were independent of the sources of recurrent activity, which he termed "M (mystery) regions". The mystery was, of course, eventually solved by the 1970's through the combination of the Parker theory of the solar wind, in-situ solar wind observations and the discovery of coronal holes. Sheeley et al. [1976, 1977] illustrate 27-day stack plots of $\mathrm{C}$, solar wind speed, and coronal holes which convincingly show the intimate relationship between recurrent geomagnetic activity, high-speed streams and coronal holes. For further discussion of the historical aspects of recurrent geomagnetic activity, see Burlaga and Lepping [1977], Cliver [1994, 1995], and references therein. In addition, Crooker and Cliver [1994] review the studies that culminated in the identification of "M-regions", and argue that the slow solar wind and CIRs should be included in this identification, in addition to corotating high-speed streams.

\section{Origin of CIR/stream associated geomagnetic activity}

Crooker [2000] notes that a "common misunderstanding about high-speed streams is that the high-speed flow itself causes geomagnetic storms". Burlaga and Lepping [1977] were among the first to examine in detail the interplanetary causes of geomagnetic activity associated with CIRs and high-speed streams and demonstrate that the magnetic field plays a prominent role. Figure 6 shows one event from their study. In addition to the solar wind density, speed and magnetic field intensity, which clearly show the stream and CIR, the figure includes the north-south component of the IMF $\left(B_{z}\right)$, the $\mathrm{y}$-component of the interplanetary electric field $\left(B_{z} V\right)$, and the geomagnetic $\mathrm{AE}$ index, which measures auroral zone activity that is not necessarily due to storms. Burlaga and Lepping [1977] noted a "striking correlation" between the bursts in AE and large southward (negative) values of $B_{z}$, concluding that " $B_{z}$ is an essential factor in causing the geomagnetic activity". The burst-like nature of $\mathrm{AE}$ results from the highly variable magnetic field on timescales of a few hours throughout the passage of the high-speed stream. These magnetic field variations in highspeed streams at both low and high heliolatitudes are predominantly large-amplitude Alfvén waves moving outward from the Sun [e.g., Belcher and Davis, 1971; Smith et al., 1995; Tsurutani et al., 1995a]. These non-compressive disturbances are perpendicular to, and propagate along, the mean magnetic field direction at the Alfvén speed, and are recognizable from correlated variations in the magnetic field and solar wind velocity components (Figure 7). Transverse fluctuations with $\triangle B /|B| \sim 1-2$ have been typically reported. Burlaga and Lepping [1977] noted that geomagnetic activity tends to be stronger in the vicinity of the CIR-associated magnetic field enhancement, where compression would be expected to enhance any southward fields present. Ulysses results support the idea that Alfvén waves moving out in high-speed streams may strengthen on entering CIRs [Tsurutani et al., 1995a].

Assessment of the geomagnetic impact of CIRs and corotating streams depends on the parameter used to measure this impact since different geomagnetic indices are dominated by activity in particular regions of the magnetosphere. For example, AE measures activity in the auroral zone, indices such as $a a, K p$, and C9 reflect mid-latitude conditions, while $D s t$, the index frequently used to indicate storm amplitudes, is dominated by the strength of the ring current. Figure 8 illustrates schematically the generation of CIR/stream related storms [Tsurutani et al., 1995b, 2006]. The figure shows first the trailing edge of a high-speed stream, where the speed is decreasing, the magnetic field intensity is low, and there are few Alfvén waves, so that geomagnetic activity is low. The higher densities associated with the heliospheric plasma sheet and the onset of compressive effects related to 
the stream-stream interaction lead to a small increase in Dst due to inward motion and intensification of the magnetopause currents. Subsequent southward deflections of the IMF result in intervals of geomagnetic activity as measured by AE. In particular, Alfvén waves in the high-speed stream lead to extended intervals of geomagnetic activity that have been termed "High Intensity Long Duration Continuous AE Activity" (HILDCAAs) [Tsurutani and Gonzalez, 1987; Tsurutani et al., 1990]. HILDCAA activity is most intense in the enhanced fields associated with the CIR. The Dst index shows a decrease to negative values, the storm main phase, associated with the CIR. Because of the fluctuating fields, the main phase may be rather irregular. Recovery occurs where the CIR field enhancement and related southward fields start to decline. Note that when the storm, as measured by Dst, has essentially recovered, auroral zone activity continues. A correlation between solar wind dynamic pressure and Dst has been reported [Murayama, 1982; Fenrich and Luhman, 1998]. Nevertheless, this parameter has a relatively weak influence on the strength of geomagnetic activity, and only at times of southward magnetic field [e.g., Smith et al., 1999].

\section{Seasonal effects}

The geoeffectiveness of CIRs/high-speed streams also depends to some extent on the time of year. The changing relative orientations of the axes of the Sun and Earth along the Earth's orbit give rise to a larger southward component of the Parker spiral field, which favors stronger activity levels, in the (northern hemisphere) autumn at times of outward IMF and in the spring for periods of sunward IMF [Russell and McPherron, 1973]. For example, Figure 9 [Sheeley et al., 1977] illustrates that the average C9 index during a number of streams in 1962 - 1975 was indeed slightly higher in those streams in which the seasonal effect was expected to be favorable (solid circles) rather than unfavorable (open circles). A seasonal effect might also be expected to result from the Earth penetrating more deeply into high-speed streams in the southern (northern) hemisphere in the spring (autumn) because of the inclination of the Earth's orbit relative to the solar equator. However, this effect will be complicated by changes associated with temporal development of the source coronal holes [e.g., Crooker et al., 1996].

\section{Long-term variations}

There are several interesting topics related to "long-term" variations in geomagnetic activity associated with CIRs and high-speed streams. These include variations in the pattern of recurrent activity from cycle to cycle, the frequency of geomagnetic storms associated with these structures as compared with those associated with solar wind transients, variations in average geomagnetic activity levels during the solar cycle, and variations on century timescales.

Figure 10 summarizes the presence of recurrent activity in the C9 index during 1950 - 2004 using a wavelet analysis [Torrence and Compo, 1998] which shows the power at periods of $10-50$ days. Each individual 10-year plot approximately encompasses a solar cycle, with solar minimum being near the center of each plot. Intermittent $\sim 1$ and $\sim$ half-solar rotation components (corresponding to one or two prominent corotating streams present) are evident. Although these components tend to be most prevalent during the declining and minimum phases of the solar cycle, the temporal pattern of power clearly varies from cycle to cycle. For example, 27-day recurrent activity was prominent at the time of the Mariner 2 observations of high-speed streams in 1962 , but was unusually weak during the remainder of the mid-1960's solar minimum, whereas it occurred during much of the next solar minimum. This analysis suggests that the pattern of recurrent activity, which presumably is dictated by the configuration of coronal holes which gives rise to the related high-speed streams, cannot simply be "predicted" from observations in previous cycles. Cliver et al. [1996] have con- 
cluded from examining the aa geomagnetic index [Mayaud, 1972] for 1844 - 1994 that recurrent activity tends to be stronger during the declining phase of even numbered solar cycles, and suggested that a more rapid equatorward expansion of coronal holes than in odd cycles may be responsible. However, this "rule" is not particularly obvious in the presentation in Figure 10, where the early 1950's, 1970's and 1990's are declining phases of even cycles.

Turning to geomagnetic storms, Figure 11 illustrates the variation in the solar wind "drivers" of geomagnetic storms during recent solar cycles [updated from Richardson et al., 2001]. Specifically, variations in the yearly numbers of "small", "medium", and "large" + "major" storms (based on the geomagnetic $K p$ index; for details see Richardson et al. [2001]) associated with CMErelated structures (ICMEs and related interplanetary shocks) and corotating streams, are shown for 1972 - 2004. The storm sizes correspond roughly to small: Dst $<\sim-50 \mathrm{nT}$; medium: Dst $<\sim-75 \mathrm{nT}$; large: Dst $<\sim-150 \mathrm{nT}$; and major: Dst $<\sim-200 \mathrm{nT}$. The sunspot number is shown at the top of the figure. Storms associated with streams are most prevalent during the decay phase of the solar cycle, but continue to be present at all phases of the cycle. These storms are generally small or medium in size. Occasional stronger stream-associated storms do occur, predominately during the declining phase of the solar cycle. Overall, we estimate that $\sim 26 \%$ of "large" storms and $\sim 3 \%$ of "major" storms in 1972 - 2004 were driven by streams, while CMEs contribute the majority of these larger storms. The occurrence rate of CME-related storms tends to follow the sunspot cycle. Nevertheless, a temporary decrease in the occurrence rate of $\geq$ medium CME-related storms is often seen near solar maximum. This is clearly present in 1979 - 1980 and 1990, while the present cycle is complicated by an additional decrease due to the temporary decrease in the ICME rate [Cane and Richardson, 2003] and prevalence of streams in 1999. The temporary reduction in the oc- currence rate of strong CME-related storms near solar maximum may be associated with the decrease in the frequency of energetic solar events around the time of the solar magnetic field reversal [e.g., Feminella et al., 1997]. Thus, the typical "double peak" in storm activity during the solar cycle [e.g., Gonzalez et al., 1990] may be the superposition of a double peak in the rate of CME-associated storms and a further peak during the declining phase associated with corotating streams, which may merge with the second CME-associated peak.

Although they are rarely responsible for major geomagnetic storms, high-speed streams do make important contributions to average geomagnetic conditions on longer $(>>$ solar rotation) timescales because of their extended duration. For example, Figure 12 [updated from Richardson et al., 2002] shows the monthly sunspot number and 3-rotation averages of the aa geomagnetic index (a mid-latitude index including both auroral zone and equatorial disturbances) averaged over all solar wind, and in CME-related solar wind, corotating high-speed streams, and slow solar wind, in 1972 - early 2005, covering $\sim 3$ solar cycles. The "all solar wind" results are repeated in the bottom three panels. Note that $a a$ is rather poorly correlated with the sunspot number $(c c=0.306)$. Reasons for this include the decrease in geomagnetic activity near sunspot maximum, which appears to reflect principally a temporary decrease in the strength of the IMF around solar field reversal, and activity during the declining phase of the solar cycle associated with high-speed streams. Overall, mean values of $a a$ at all solar activity levels closely track those found in high-speed streams. Thus, long-term averages in $a a$ are determined predominantly by activity levels in the background (nonCME-related) solar wind, in particular associated with high-speed streams.

The $a a$ index is of particular interest because it extends back to the middle of the $19^{\text {th }}$ century, as mentioned above. An interesting feature of this index is that it shows a clear increase dur- 
ing the first half of the 20th century at all activity levels [Vennestroem, 2000]. Although there is some debate about this feature [e.g., Svalgaard et al., 2004], a possible interpretation is that the solar wind magnetic field strength increased during this period, raising the general level of geomagnetic activity [Lockwood et al., 1999]. Figure 13 [Richardson et al., 2002] compares the aa index during the solar minimum years of 1977 and 1901 (shaded), and clearly shows the lower geomagnetic activity levels at the beginning of the $20^{\text {th }}$ century. Note that recurrent geomagnetic activity did occur in 1901 (examples are indicated by arrowheads), suggesting that stream interactions and hence variations in solar wind speed, were present at this time.

\section{ICME-CIR interactions}

We finally briefly mention an interesting situation that occurs occasionally [e.g., Zhao, 1992; Cane and Richardson, 1997, Fenrich and Luhmann, 1998; Crooker, 2000] and has been modeled by Odstrcil and Pizzo [1999]. This is when a high-speed stream interacts with, and compresses the trailing edge of, a preceding, slower ICME. If the magnetic field inside the compressed region of the ICME includes a southward component, interaction with the high-speed stream may enhance the geoeffectiveness of the ICME. Some "unexpectedly strong" geomagnetic storms have resulted from this configuration. An example in October, 1999 is shown in Figure 14. The probable ICME [Cane and Richardson, 2003] is bounded by dashed vertical lines (the upstream shock is indicated by the solid line). The trailing edge evidently formed the interface with the following high-speed stream. The interaction apparently enhanced the region of southward magnetic field inside the trailing edge, resulting in the major geomagnetic storm indicated by the Dst and $\mathrm{Kp}$ indices. The storm then decayed within the high-speed flow where the field was weaker and variable in direction.

\section{Summary}

Corotating streams, and the CIRs formed at their leading edges by stream-stream interaction, are typically associated with modest levels of geomagnetic activity that recurs at the solar rotation period. The principal causes appear to be intermittent, brief intervals of southward magnetic field resulting from field fluctuations associated with Alfvén waves propagating away from the Sun. These may be amplified (together with their possible geomagnetic effects) when propagating into the CIR. CIRs and streams are typically associated with weak geomagnetic storms, but occasionally with significant storms, most frequently during the declining phase of the solar cycle. Unexpectedly strong storms may also result when a stream interacts with, and compresses, an ICME with an embedded southward field. Longer-term studies suggest that the pattern of recurrent geomagnetic activity varies from cycle-to-cycle, streams are a major contributor to average geomagnetic activity levels, and that recurrent activity, and hence presumably streams of fast and slower solar wind were present at the beginning of the 20th century, when geomagnetic activity levels appear to have been generally lower than during the last $\sim 50$ years.

Acknowledgments. The meeting organizers are thanked for the opportunity to prepare this review, and for assistance with travel to Manaus. The use of ACE data, available from the ACE Science Center, and data from the National Space Science Data Center and National Geophysical Data Center, is gratefully acknowledged.

\section{References}

Balngh, A., R. G. Marsden, and E. J. Smith (eds.), The Heliosphere Near Solar Minimum - The Ulysses Perspective, Springer-Verlag, Berlin, Heidelberg, New York, 2001

Bartels, J., Terrestrial magnetic activity and its relation to solar phenomena, Terr. Magn. Atmos. Elect., 37, 1, 1932. 
Bartels, J., Solar activity and geomagnetism, Terr. Magn. Atmos. Elect., 45, 339, 1940.

Belcher, J. W., and L. Davis, Large amplitude Alfvén waves in the interplanetary medium, 2, J. Geophys. Res., 76, 3534, 1971.

Berdichevsky, D. B., A. Szabo, R. P. Lepping, A. F. Viñas, and F. Mariani, Interplanetary fast shocks and associated drivers observed through the $23^{\text {rd }}$ solar minimum by Wind over its first 2.5 years, J. Geophys. Res., 105, 27,289, 2000.

Bravo, S., G. A. Stewart, and X. Blanco-Cano, The varying multipolar structure of the Sun's magnetic field and the evolution of the solar magnetosphere through the solar cycle, Solar Phys., 179, 223, 1998.

Burlaga, L. F., A reverse hydromagnetic shock in the solar wind, Cosmic Electrodyn., 1, 233, 1970.

Burlaga, L. F., Interplanetary stream interfaces, J. Geophys. Res., 79, 3717, 1974.

Burlaga, L. F., Interplanetary Magnetodynamics, Oxford University Press, New York and Oxford, 1995.

Burlaga, L. F., and R. P. Lepping, The causes of recurrent geomagnetic storms, Planet. Space Sci., 25, 1151, 1977.

Burlaga, L. F., R. Schwenn, R., and H. Rosenbauer, Dynamical evolution of interplanetary magnetic fields and flows between $0.3 \mathrm{AU}$ and 8.5 AU: Entrainment, Geophys. Res. Lett., 10, 413, 1983.

Cane, H. V., and I. G. Richardson, What caused the large geomagnetic storm of November 1978?, J. Geophys. Res., 102, 17, 445, 1997.

Cane, H. V., and I. G. Richardson, Interplanetary coronal mass ejections in the near-earth solar wind during 1996-2002, J. Geophys. Res., 108(4), 10.1029/2002JA009817, 2003.

Chao, J. K., V. Formisano, and P. C. Hedgecock, Shock pair observation, in C. P. Sonett, P. J. Coleman, Jr, and J. M. Wilcox (eds.), Solar Wind, NASA SP-308, p. 435, 1972.
Cliver, E. W., Solar activity and geomagnetic storms: The corpuscular hypothesis, Eos Trans. AGU, 75(52) 609, 1994.

Cliver, E. W., Solar activity and geomagnetic storms: From $\mathrm{M}$ regions and flares to coronal holes and CMEs, Eos Trans. AGU, 76(8) 75, 1995.

Cliver, E. W., V. Boriakoff, and K. H. Bounar, The 22-year cycle of geomagnetic and solar wind activity, J. Geophys. Res., 101, 27,091, 1996.

Crooker, N. U., Solar and heliospheric geomagnetic disturbances, J. Atmosph. Solar Terr. Phys., 62, 1071, 2000.

Crooker, N. U., and E. W. Cliver, Postmodern view of M-regions, J. Geophys. Res., 99, 23,383, 1994.

Crooker, N. U., et al., A two-stream, four-sector, recurrence pattern: Implications from WIND for the 22-year geomagnetic activity cycle, $J$. Geophys. Res., 23, 1275, 1996.

Crooker, N. U., et al., CIR Morphology, turbulence, discontinuities and energetic particles: Report of Working Group 2, Space Sci. Rev. 89, 179, 1999.

Dungey, J. W., Interplanetary magnetic field and the auroral zones, Phys. Rev. Lett., 6, 47, 1961.

Feminella, F., and M. Storini, Large scale dynamical phenomena during solar activity cycles, Astron. Astrophys., 322, 311, 1997.

Fenrich, F. R., and J. G. Luhmann, Geomagnetic response to magnetic clouds of different polarity, Geophys. Res. Lett., 25, 2999, 1998.

Forsyth, R. J., and E. Marsch, Solar origin and interplanetary evolution of stream interfaces, Space Sci. Rev., 89, 7, 1999.

Gazis, P. R.: 1984, Observations of plasma bulk parameters and the energy balance between 1 and $10 \mathrm{AU}$, J. Geophys. Res., 89, 775, 1984.

Gazis, P. R., and A. J. Lazarus, The radial evolution of the solar wind, $1-10 \mathrm{AU}$, in M. Neugebauer(ed.), Solar Wind Five, NASA 
Conf. Publ. 2280, Washington D.C., p. 509, 1983.

Gosling, J. T., Corotating and transient solar wind flows in three dimensions, Ann. Rev. Astron. Astrophys., 34, 35, 1996.

Gosling, J. T., and V. J. Pizzo, Formation and evolution of corotating interaction regions and their three dimensional structure, Space Sci. Rev., 89, 21, 1999.

Gosling, J. T., A. J.,Hundhausen, and S. J. Bame, Solar wind evolution at large heliocentric distances: Experimental demonstration and test of a model, J. Geophys. Res., 81, 2111, 1976.

Gosling, J. T., J. R. Asbridge, S. J. Bame, and W. C. Feldman, Solar Wind Stream Interfaces, J. Geophys. Res., 83, 1401, 1978.

Gosling, J. T., D. J. McComas, J. L. Phillips, and S. J. Bame, Geomagnetic activity associated with Earth passage of interplanetary shock disturbances and coronal mass ejections, $J$. Geophys. Res., 96, 7831, 1991.

Gosling, J. T., et al., Latitudinal variation of solar wind corotating stream interaction regions, Geophys. Res. Lett., 20, 2789, 1993.

Greaves, W. M. H., and H. W. Newton, On the recurrence of magnetic storms, Mon. Not. $R$. Astron. Soc., 89, 641, 1929.

Hundhausen, A. J., Nonlinear model of highspeed solar wind streams, J. Geophys. Res., 78, 1528, 1973a.

Hundhausen, A. J., Evolution of large-scale solar wind structures beyond $1 \mathrm{AU}$, J. Geophys. Res. 78, 2035-2042,1973b.

Hundhausen, A. J., and J. T. Gosling, Solar wind structure at large heliocentric distances: An interpretation of Pioneer 10 observations, $J$. Geophys. Res., 81, 1436, 1976.

Intriligator, D. S., and G. L. Siscoe, Stream interfaces and energetic ions closer than expected: Analyzes of Pioneers 10 and 11 observations, Geophys. Res. Lett., 21, 1117, 1994.

Krieger, A. S., A. F. Timothy, and E. C. Roelof, A coronal hole and its identification as the source of a high velocity solar wind stream, Sol. Phys., 29, 505, 1973.

Lazarus, A. J., K. W. Ogilvie, and L. F. Burlaga, Interplanetary shock observations made by Mariner 2 and Explorer 34, Solar Phys., 13, 232, 1970.

Lockwood, M., R. Stamper, and N. M. Wild, A doubling of the sun's coronal magnetic field during the past 100 years, Nature, 399, 437, 1999.

Luhmann, J. G., Y. Li, C. N. Arge, P. Gazis, and R. Ulrich, Solar cycle changes in coronal holes and space weather cycles, J. Geophys. Res., 107, 10.1029/2001JA007550, 2002.

Maunder, E. W., Magnetic disturbances, 1882 to 1903 , as recorded at the Royal Observatory, Greenwich, and their association with sunspots, Mon. Not. R. Astron. Soc. London, 65, 2, 1905.

Mayaud, P. N., The aa indices: a 100-year series characterising the geomagnetic activity, $J$. Geophys. Res., 72, 6870, 1972.

McComas, D. J. et al., Solar wind observations over Ulysses first full polar orbit, J. Geophys. Res., 105, 10,419, 2000.

McComas, D. J., R. Goldstein, J. T. Gosling, and R. M. Skoug, Ulysses second orbit: Remarkably different solar wind, Space Sci. Rev., 97, 99, 2001.

McComas, D. J., H. A. Elliott, N. A. Schwadron, J. T. Gosling, R. M. Skoug, and B. E. Goldstein, The three-dimensional solar wind around solar maximum, Geophys. Res. Lett., 30, 1517, doi:10.1029/2003GL017136, 2003.

Murayama, T., Coupling function between solar wind parameters and geomagnetic indices, Rev. Geophys. and Space Phys., 20, 623, 1982.

Neugebauer, M. and C. W. Snyder, Mariner 2 observations of the solar wind, 1: Average properties, J. Geophys. Res., 71, 4469, 1966.

Neugebauer, M., P. C. Liewer, E. J. Smith, R. M. Skoug, and T. H. Zurbuchen, Sources of the solar wind at solar activity maximum, J. Geophys. Res., 10\%, 10.1029/2001JA000306, 2002. 
Odstrcil, D., and V. J. Pizzo, Three-dimensional propagation of coronal mass ejections (CMEs) in a structured solar wind flow, 1. CME launched within the streamer belt, J. Geophys. Res., 104, 483, 1999.

Parker, E. N., Dynamics of the Interplanetary Gas and Magnetic Fields, Astrophys. J., 128, 664, 1958.

Parker, E. N., Interplanetary Dynamical Processes, John Wiley, New York, 1963.

Perreault, P., and S.-I. Akasofu. A study of geomagnetic storms, Geophys. J. R. Astr. Soc., 54, 547, 1978.

Pizzo, V. J., and J. T. Gosling, Threedimensional simulation of high-latitude interaction regions: Comparison with Ulysses results, Geophys. Res. Lett., 21, 2063, 1994.

Richardson, I. G., Energetic particles and corotating interaction regions in the solar wind, Space Sci. Rev., 111, 267, 2004.

Richardson, I. G., and R. D. Zwickl, Low energy ions in corotating interaction regions at 1 AU: Observations, Planet. Space Sci., 32, 1179, 1984.

Richardson, I. G., G. Wibberenz, and H. V. Cane, The relationship between recurring cosmic ray depressions and corotating solar wind streams at $\leq 1$ AU: IMP 8 and Helios 1 and 2 anticoincidence guard rate observations, $J$. Geophys. Res. 101, 13 483, 1996.

Richardson, I. G., H. V. Cane, and E. W. Cliver, Sources of geomagnetic storms for solar minimum and maximum conditions during $1972-$ 2000, Geophys. Res. Lett., 28, 2569, 2001.

Richardson, I. G., H. V. Cane, and E. W. Cliver, Sources of geomagnetic activity during nearly three solar cycles (1972-2000), J. Geophys. Res., 107, 10.1029/2001JA000504, 2002.

Rickett, B. J., and W. A. Coles, Evolution of the solar wind structure over a solar cycle: Interplanetary scintillation velocity measurements compared with coronal observations, J. Geophys. Res., 96, 1717, 1991.
Riley, P., J. T. Gosling, L. A. Weiss, L. A., and V. J. Pizzo, The tilts of corotating interaction regions at midheliographic latitudes, J. Geophys. Res., 101, 24,349, 1996.

Schwenn, R., Large-scale structure of the interplanetary medium, in R. Schwenn and E. Marsch(eds.), Physics of the Inner Heliosphere, Vol. 1, p. 99, Springer-Verlag, Berlin, Heidelberg, New York, 1990.

Schwenn, R., and E. Marsch (eds.), Physics of the Inner Heliosphere, Springer-Verlag, Berlin, Heidelberg, New York, 1990.

Sheeley, N. R., Jr., J. W. Harvey, and W. C. Feldman, Coronal holes, solar wind streams, and recurrent geomagnetic disturbances, 1973 - 1976, Solar Phys., 49, 271, 1976.

Sheeley, N. R., Jr., J. S. Asbridge, S. J. Bame, and J. W. Harvey, A pictorial comparison of interplanetary magnetic field polarity, solar wind speed, and geomagnetic disturbance index during the sunspot cycle, Solar Phys., 52, 485, 1977.

Smith, E. J., and J. H. Wolfe, Observations of interaction regions and co-rotating shocks between one and five AU: Pioneer 10 and 11, Geophys. Res. Lett., 3, 137, 1976.

Smith, E. J., and J. H. Wolfe, Pioneer 10 and 11 Observations of evolving solar wind streams and shocks beyond $1 \mathrm{AU}$, in M. Shea, D. F. Smart, and S. T. Wu (eds.), Study of Traveling Interplanetary Phenomena, D. Riedel, Hingham, MA, p. 227, 1977.

Smith, E. J., and J. H. Wolfe, Fields and plasmas in the outer solar system, Space Sci. Rev., 23, 217, 1979.

Smith, E. J., A. Balogh, M. Neugebauer, and D. McComas, Ulysses observations of Alfvén waves in the southern and northern solar hemispheres, Geophys Res. Lett., 22, 3381, 1995.

Smith, J. P., M. F. Thomsen, J. E. Borovsky, and M. Collier, Solar wind density as a driver for the ring current in mild storms, Geophys. Res. Lett., 26, 1797, 1999. 
Svalgaard, L., E. W. Cliver, and P. Le Sager, IHV: a new long-term geomagnetic index, Adv. Space Res., 34(2), 436, 2004.

Torrence, C., and G. P. Compo, A practical guide to wavelet analysis, Bull. Am. Meteorol. Soc., 79, 61, 1998.

Thomas, B. T. and E. J. Smith, The structure and dynamics of the heliospheric current sheet, J. Geophys. Res., 86, 11,105, 1981.

Tsurutani, B. T., and W. D. Gonzalez, The cause of high intensity long-duration continuous $\mathrm{AE}$ activity (HILDCAAs); Interplanetary Alfvén wave trains, Planet. Space Sci., 35, 405, 1987.

Tsurutani, B. T., and W. D. Gonzalez, The interplanetary causes of magnetic storms: A review, in B. T. Tsurutani, W. D. Gonzalez, Y. Kamide, and J. K. Arballo (eds.), Magnetic Storms, Geophys. Monogr., 98, AGU, Washington, D.C., p. 77, 1997.

Tsurutani, B. T., T. Gould, B. E. Goldstein, W. D. Gonzalez, and M. Sugiura, Interplanetary Alfvén waves and auroral (substorm) activity: IMP 8, J. Geophys. Res., 95, 2241, 1990.

Tsurutani, B. T., C. M. Ho, J. K. Araballo, B. E. Goldstein, and A. Balogh, Large amplitude IMF fluctuations in corotating interaction regions: Ulysses at midlatitudes, Geophys. Res. Lett., 22, 3397, 1995a.

Tsurutani, B. T., W. D. Gonzalez, A. L. C. Gonzalez, F. Tang, J. K. Araballo, and M. Okada, Interplanetary origin of geomagnetic activity in the declining phase of the solar cycle, $J$. Geophys. Res., 100, 21,717, 1995b.

Tsurutani, B. T., N. Gopalswamy, R. L. McPherron, W. D. Gonzalez, G. Lu, and F. L. Guarnieri, Magnetic storms caused by corotating solar wind steams, this volume, 2006.

Vennerstroem, S., Long-term rise in geomagnetic activity: A close connection between quiet days and storms, Geophys. Res. Lett., 27, 69, 2000.

Wang, Y.-M., and N. R. Sheeley, Jr., The solar wind and its magnetic sources at sunspot maximum, Astrophys. J., 587, 818, 2003.
Wilcox, J., and N. Ness, Quasi-stationary corotating structure in the interplanetary medium, J. Geophys. Res., 70, 5793, 1965.

Wimmer-Schweingruber, R. F., R. von Steiger, and R. Paerli, Solar wind stream interfaces in corotating interaction regions: SWICS/Ulysses results, J. Geophys. Res., 102, 17,407, 1997.

Zhao, X., Interaction of fast steady flow with slow transient flow: A new cause of shock pair and interplanetary $B_{z}$ event, J. Geophys. Res, 97, 15,051, 1992.

Zirker, J. B. (ed.), Coronal Holes and High Speed Wind Streams, Skylab Solar Workshop, Colorado University Press, Boulder, CO, 1977.

I. G. Richardson, Laboratory for High Energy Astrophysics, Code 661, NASA Goddard Space Flight Center, Greenbelt, MD, 20771 (ian.richardson@gsfc.nasa.gov)

Figure 1. Schematic of two high-speed streams corotatir Belcher and Davis, 1971]. Variations in plasma parameter

Figure 2. Coronal holes observed by the SOHO EIT ins1 the SOHO/EIT consortium). (a) An equatorward extensio 2003); (b) a near-equatorial coronal hole (December 31, 1 in Figure 3.

Figure 3. ACE magnetic field and solar wind plasma p. rate of the IMP 8 GME anticoincidence guard) for a 27 when three corotating high-speed streams with CIRs at th lines indicate stream interface passages.

Figure 4. Energetic particle, solar wind plasma and ma: the same CIR at $1 \mathrm{AU}$, Pioneer 11 at $3.8 \mathrm{AU}$, and Pioneer 2004]. Forward $(F)$ and reverse $(R)$ shocks and stream panels show magnetic field turbulence levels.

Figure 5. 27-day (Bartels rotation) stackplot of the daily number (R9) for 1971 - 1974.

This preprint was prepared with AGU's IATEX macros v4. File CIRmanaus6 formatted September 26, 2005. 
Fi६ STACK̂: A high-speed stream in November 1973 showing the relationship between bursts of AE activity and brief southward (negative) turnings of the IMF throughout passage of the stream [Burlaga and Lepping, 1977].

Figure 7. Examples of Alfvén waves [Belcher and Davis, 1971], showing correlated variations in the three components of the IMF and solar wind speed (in RTN coordinates).

Figure 8. Schematic of the generation of geomagnetic activity by Alfven waves in high-speed streams, showing the different temporal profiles of the AE and Dst geomagnetic indices (Tsurutani et al. [2006], updated from Tsurutani et al. [1995b]). The vertical dashed line indicates a representative location for the heliospheric current sheet crossing, if present.

Figure 9. Average C9 index and solar wind speed in a group of high-speed streams observed in spring or fall, showing higher geomagnetic activity levels in streams of similar speeds with favorably-oriented IMFs [Sheeley et al., 1977].

Figure 10. Wavelet analysis of the C9 index for 1950 - 2004, showing power (arbitrary units) at periods of 10 - 50 days versus time. Each 10-year panel approximately encompasses a solar cycle, with solar minimum near the cen 


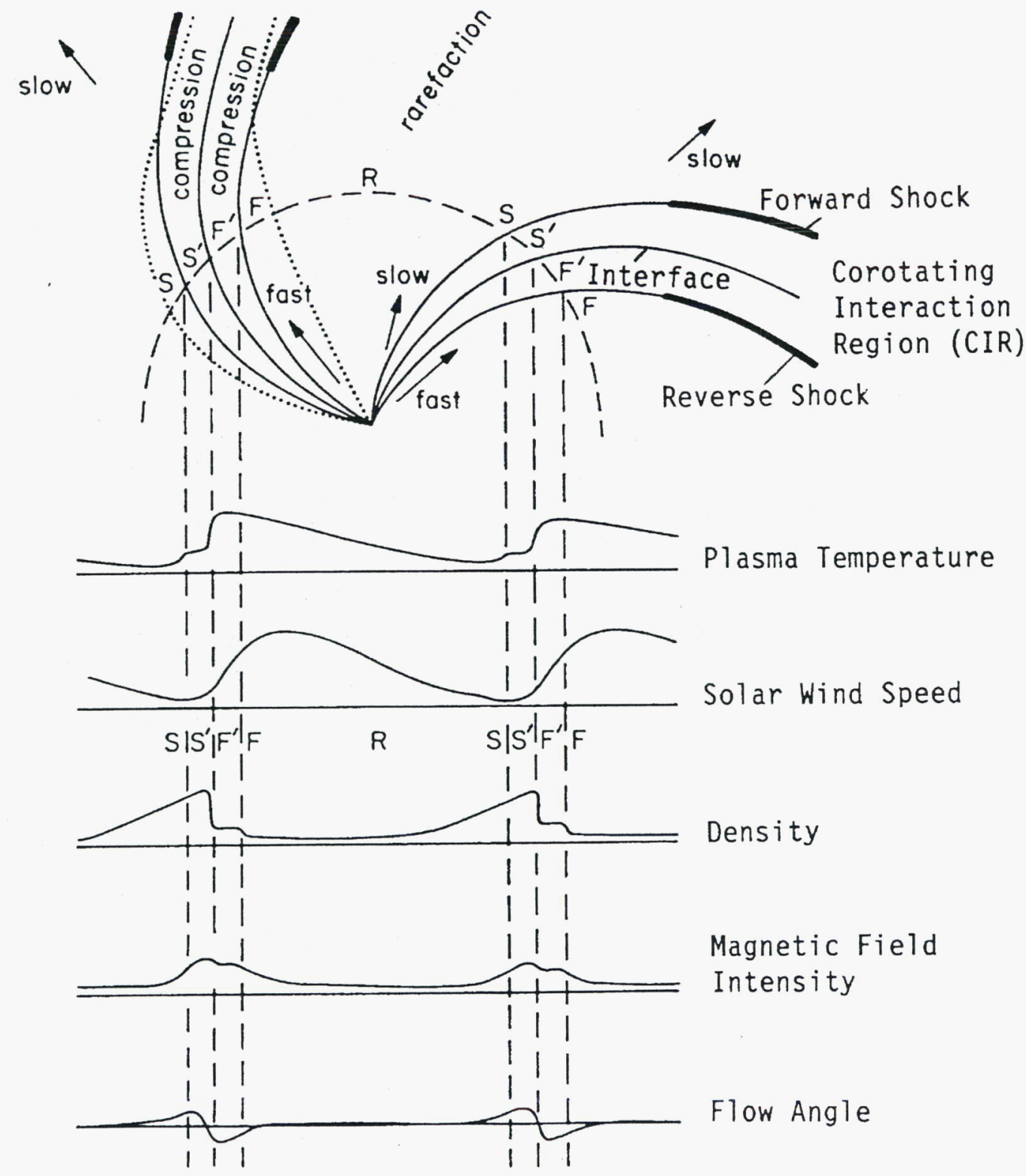



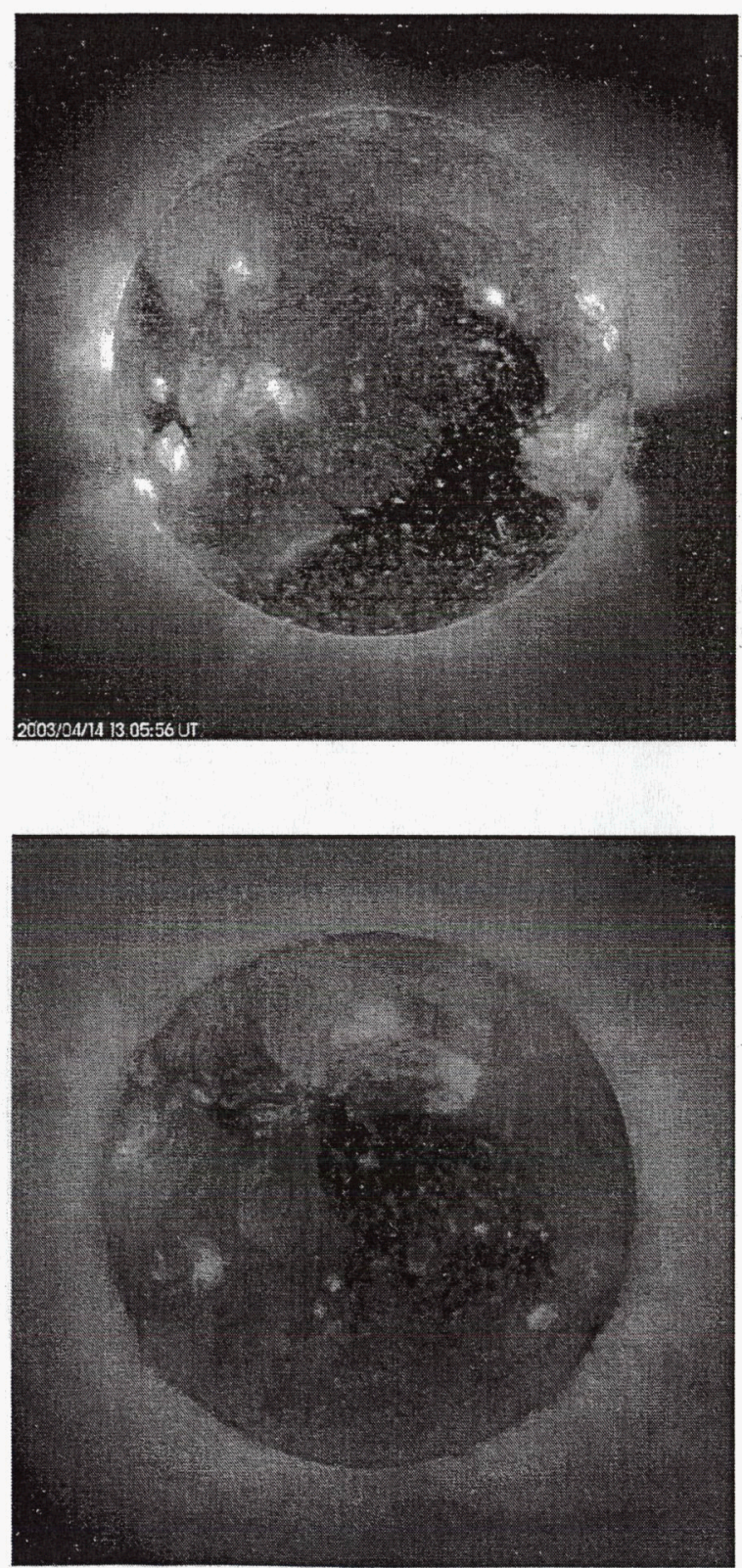


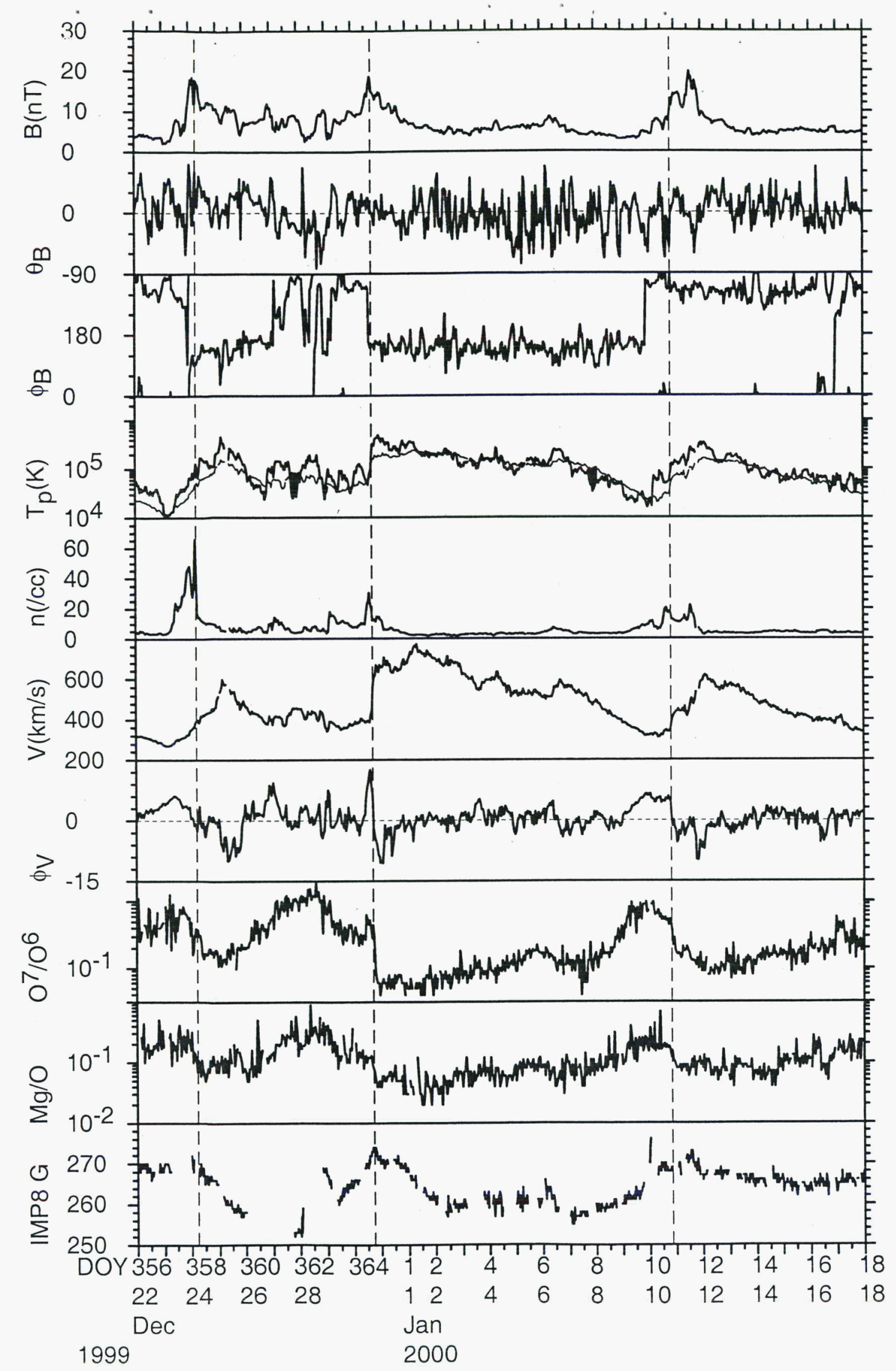



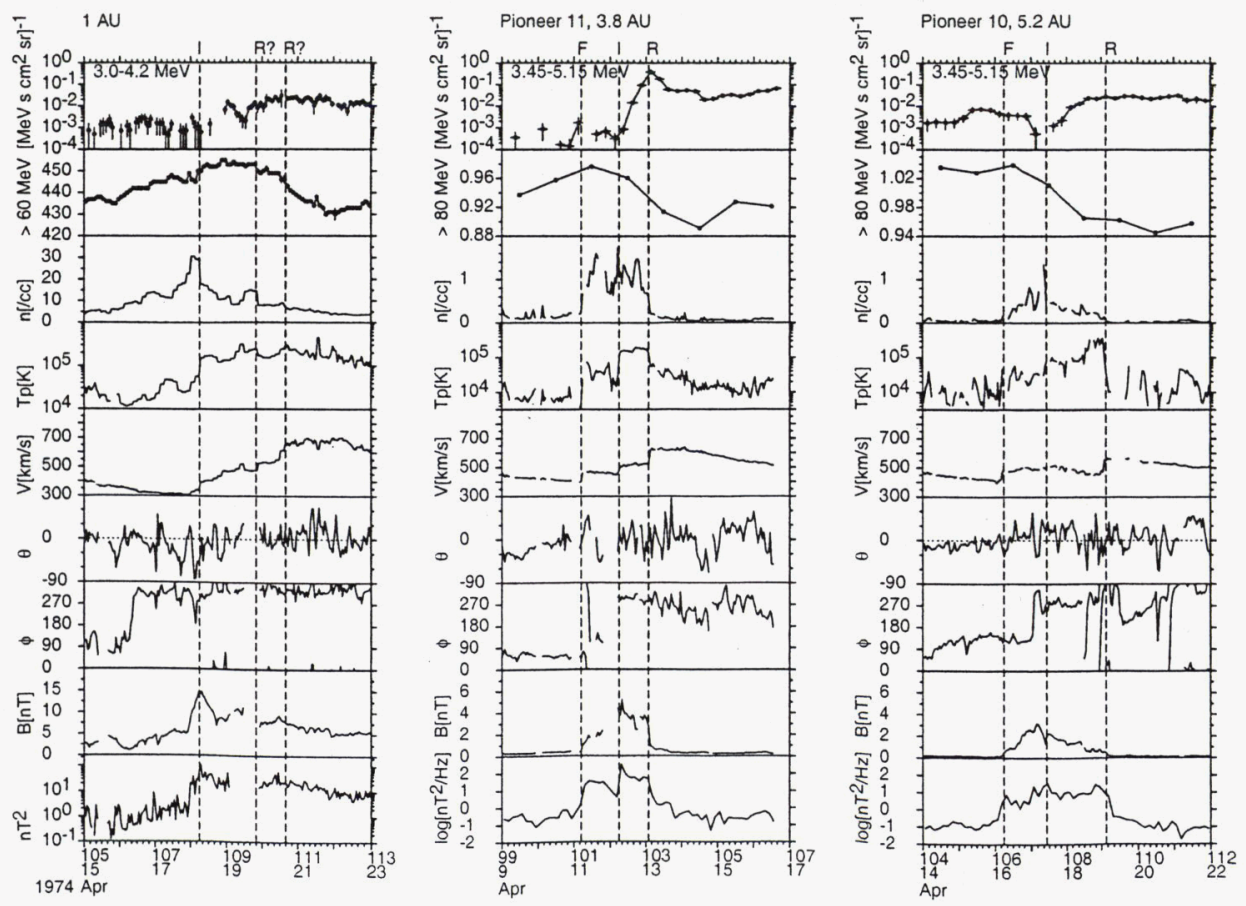


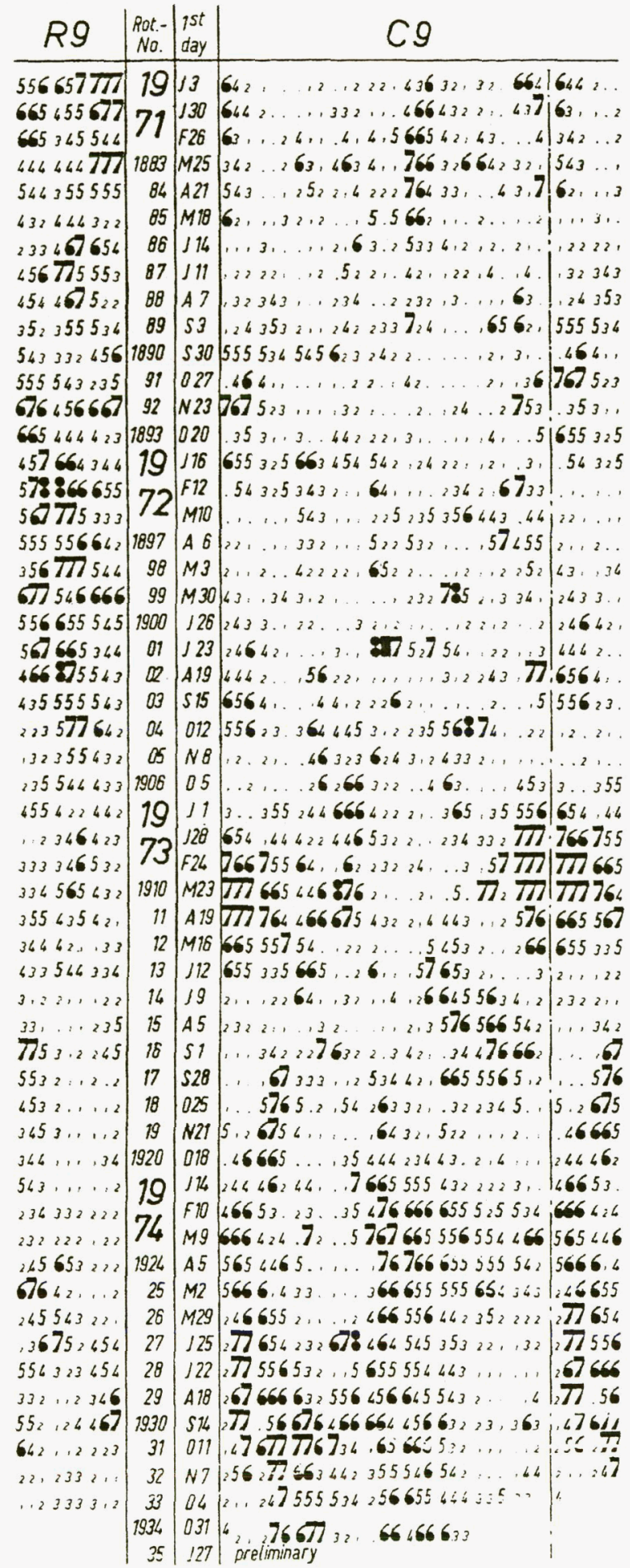




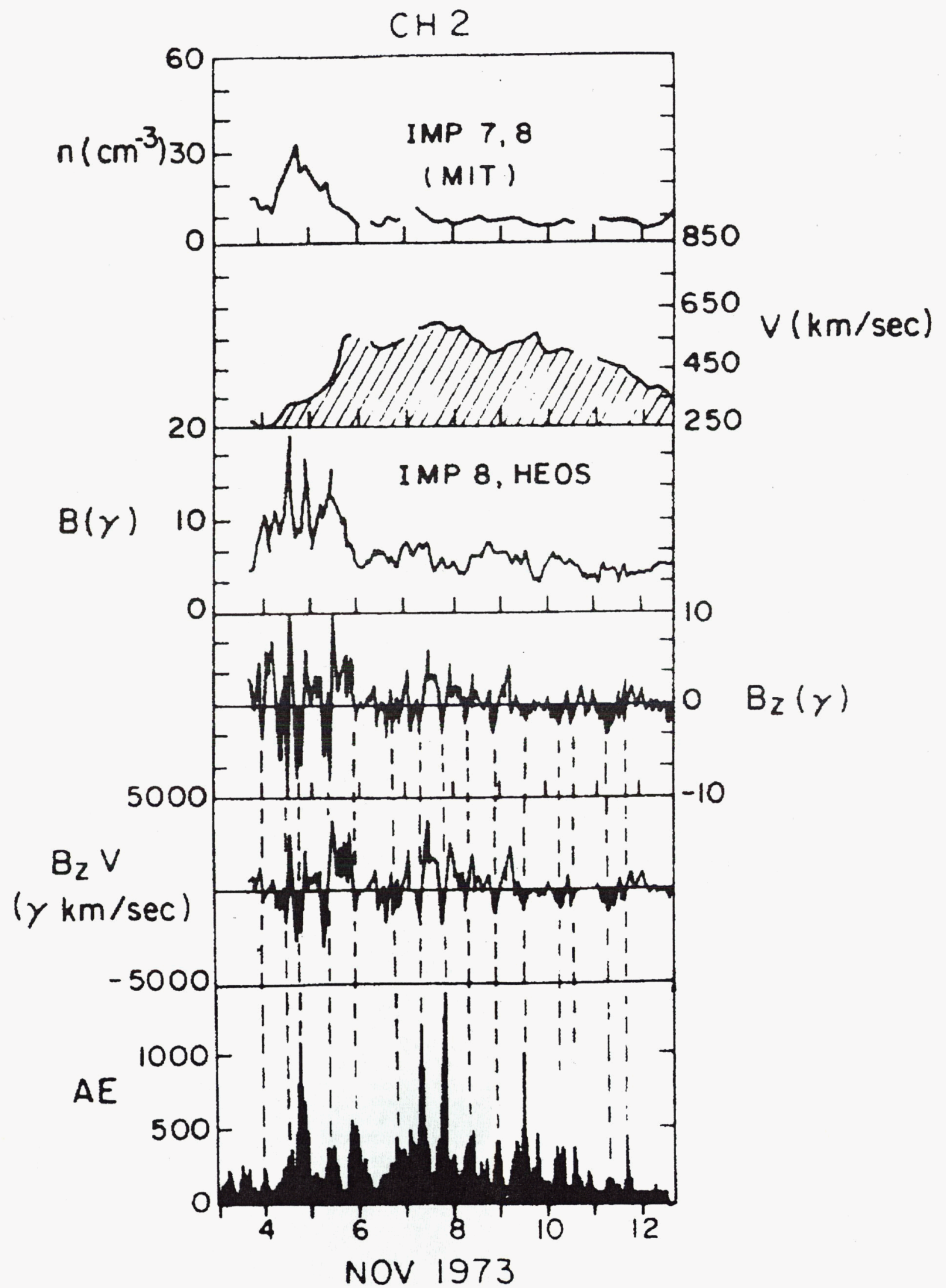




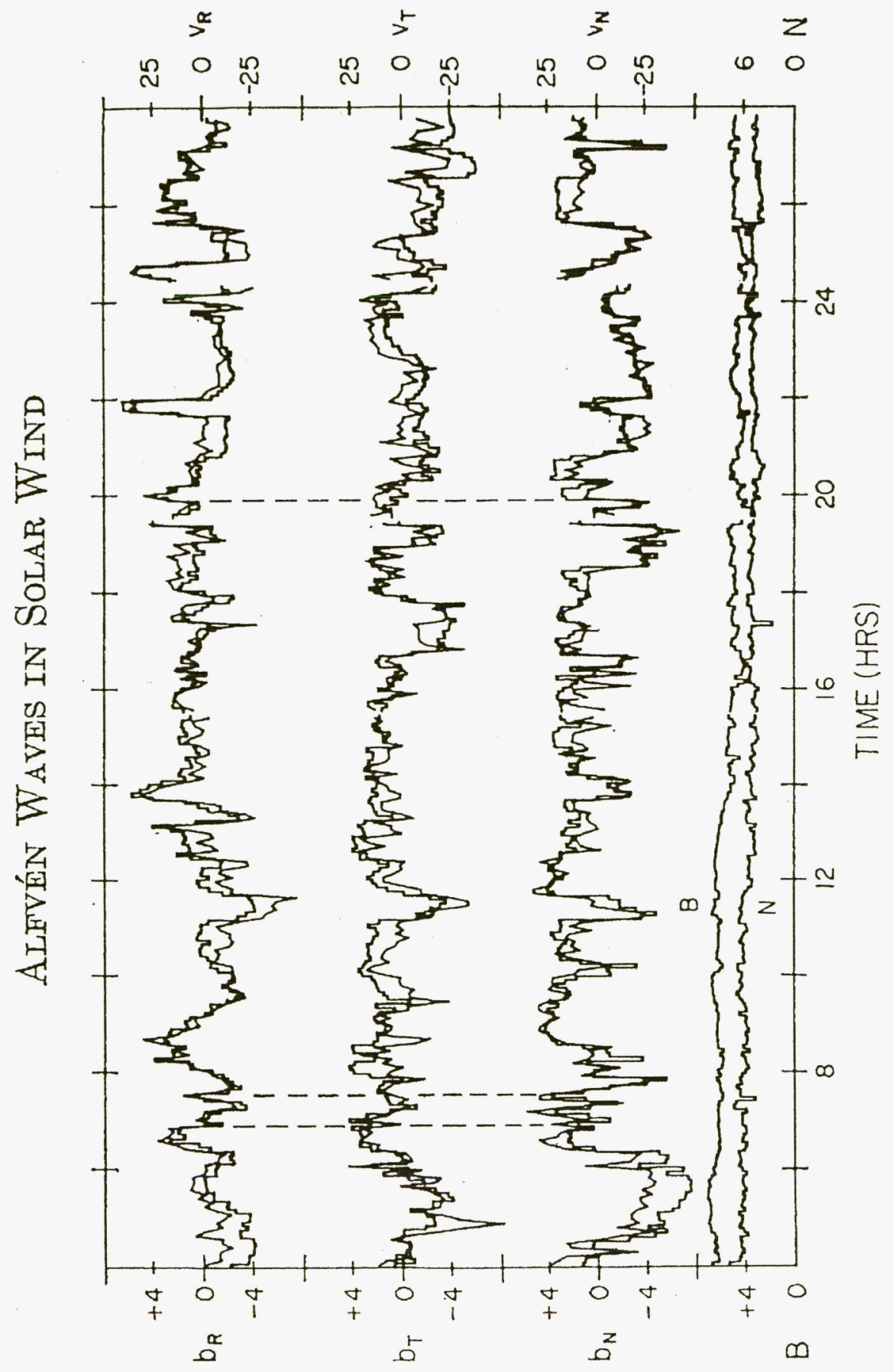




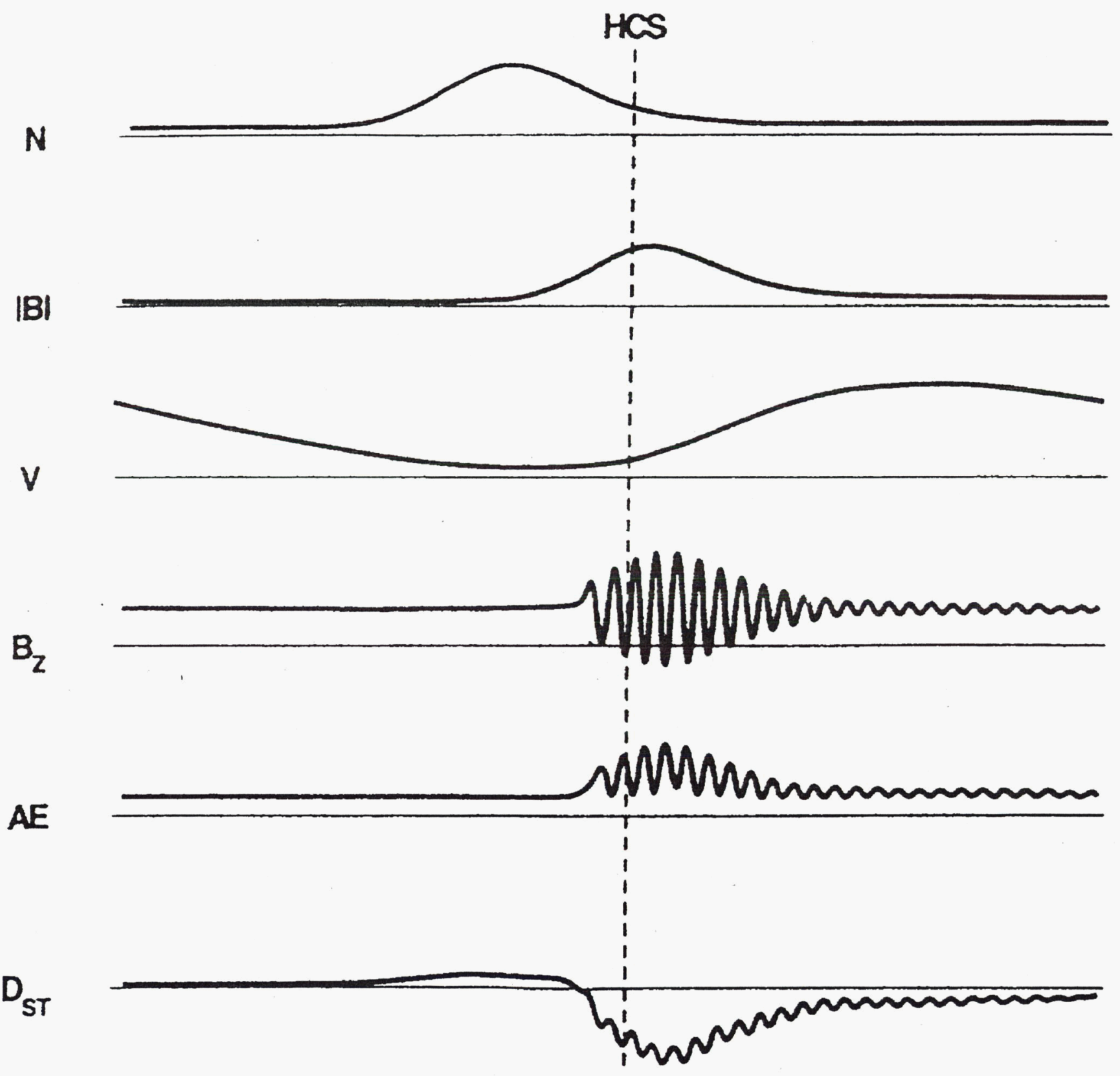




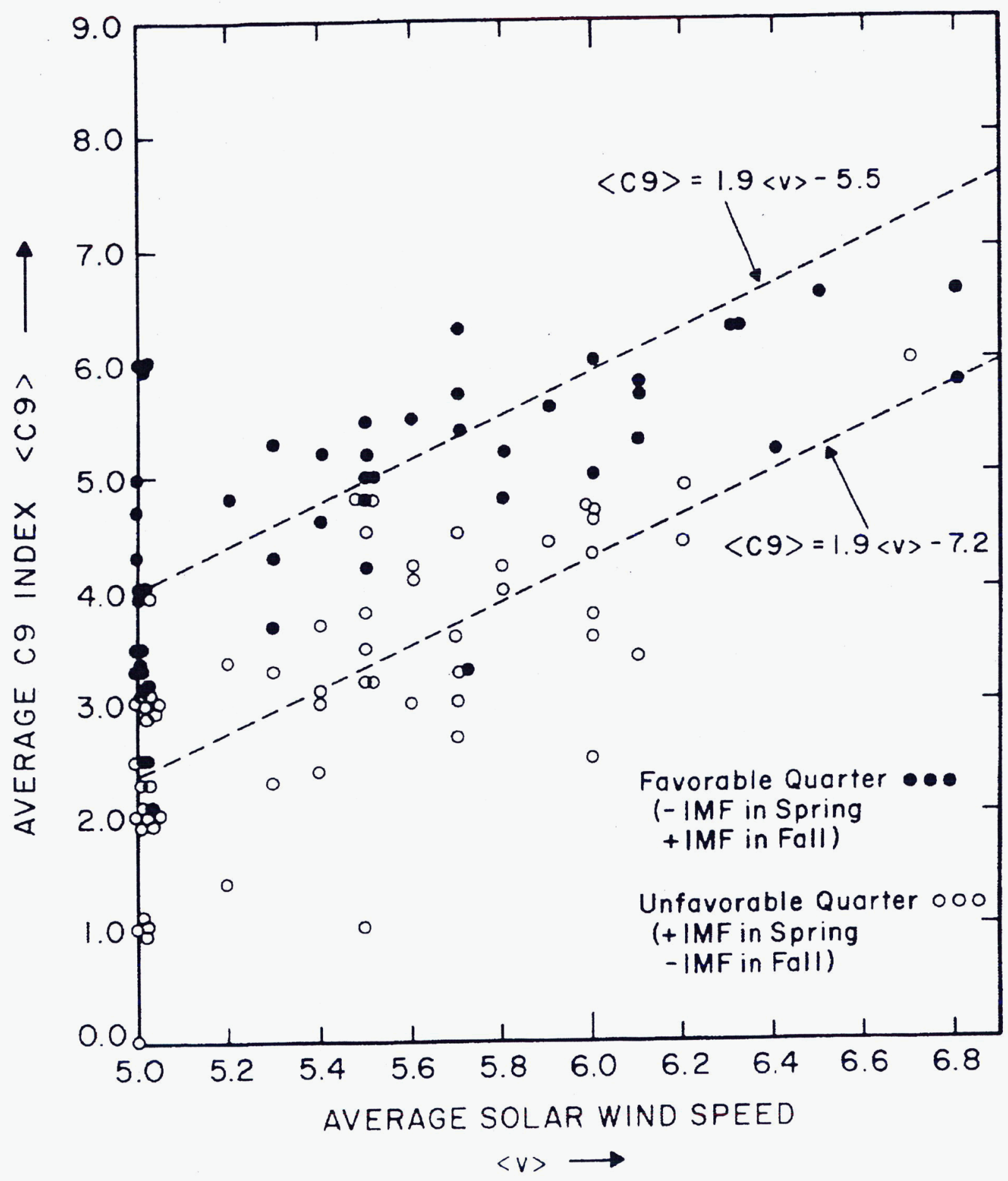



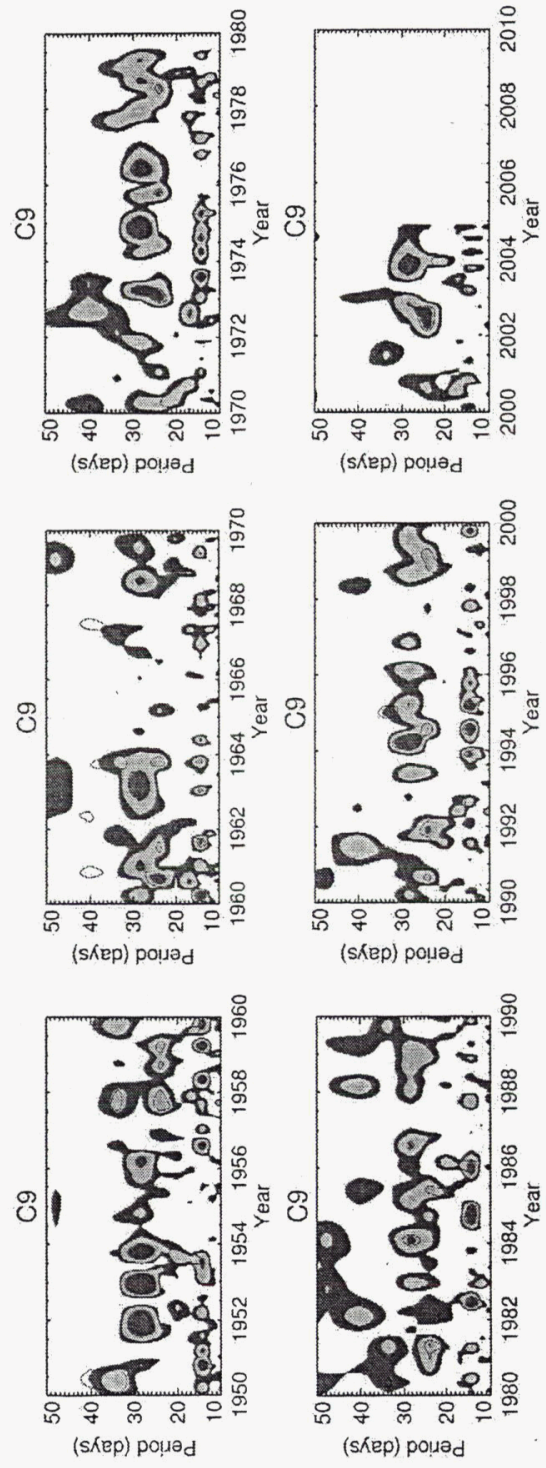


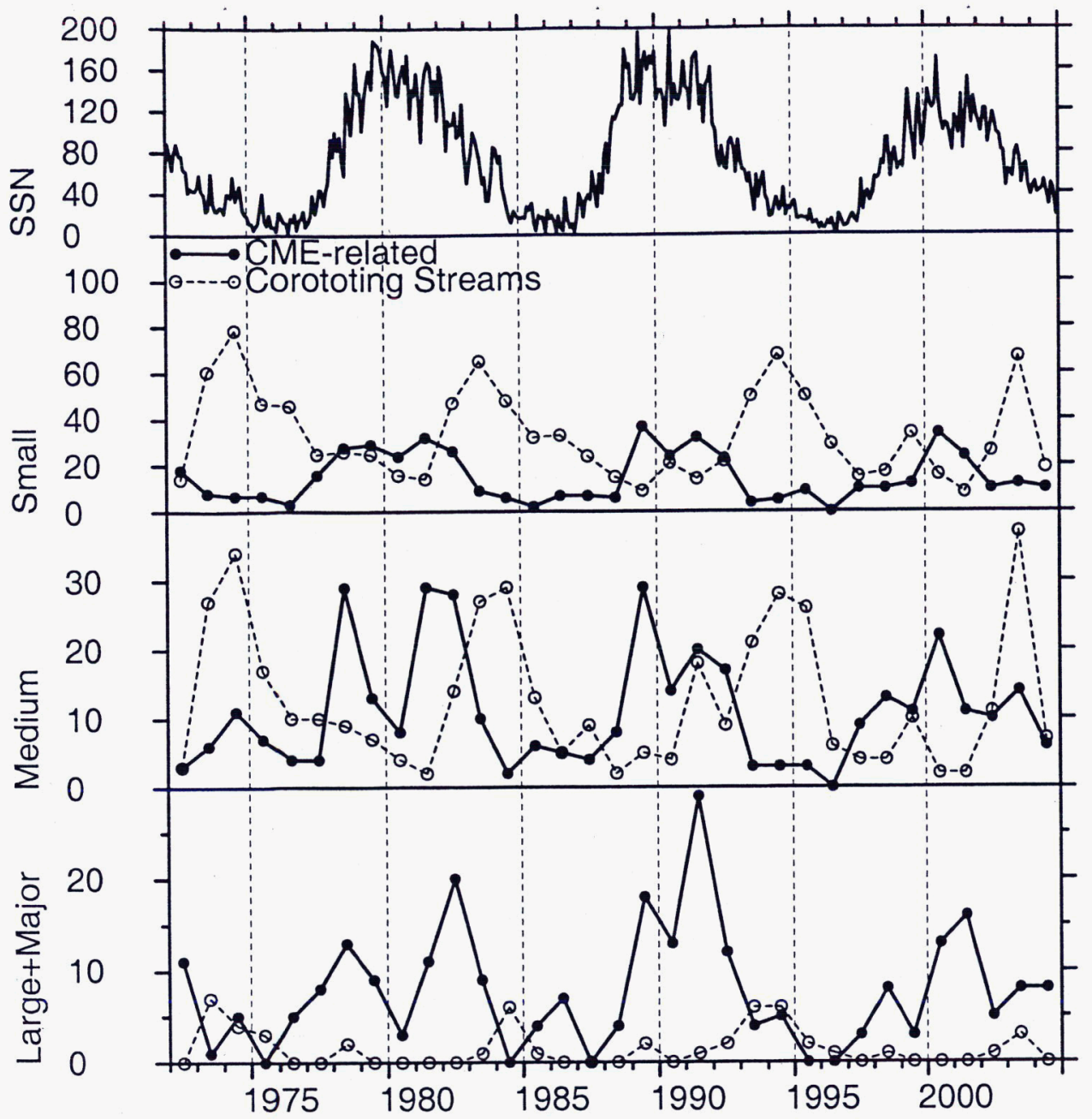




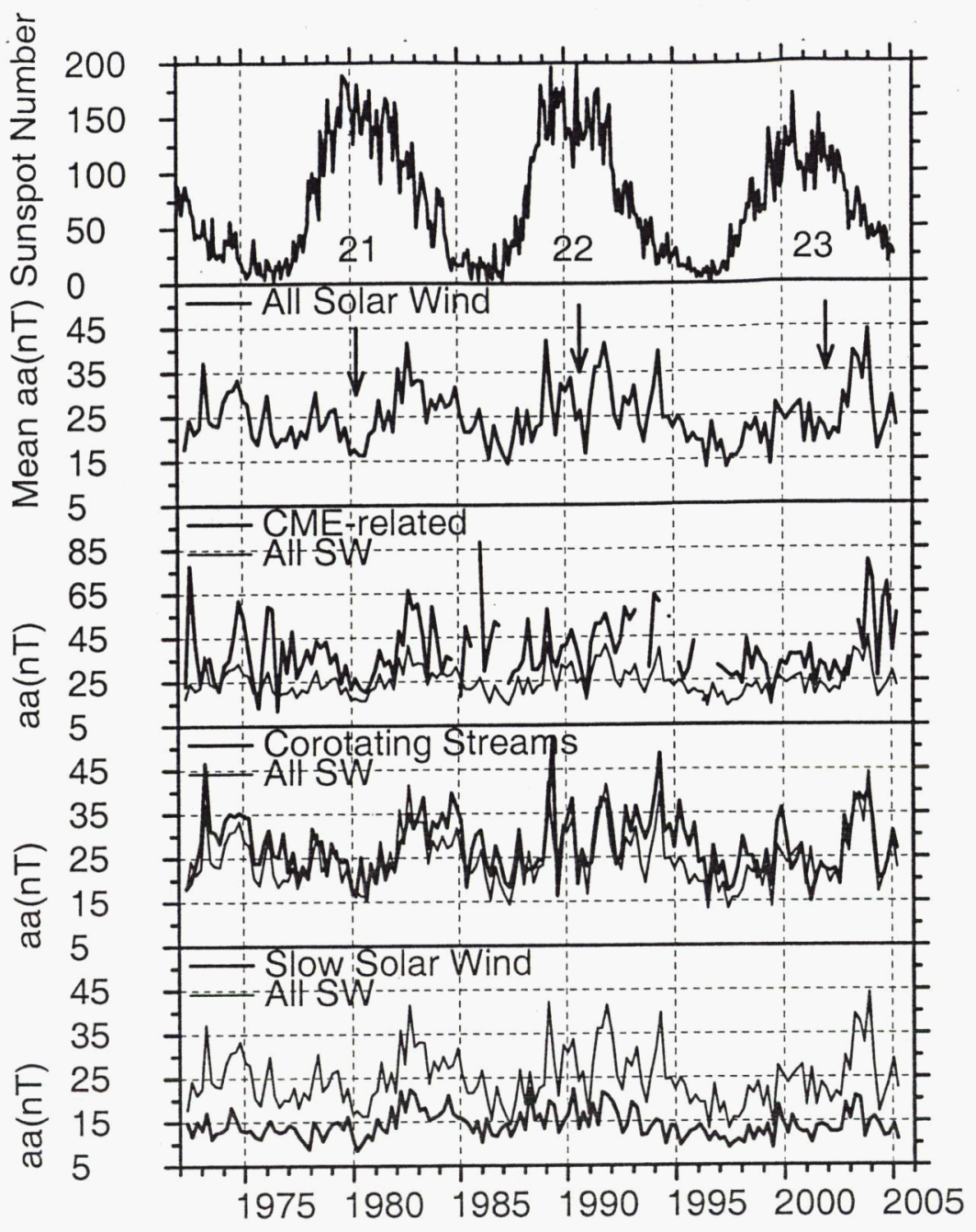




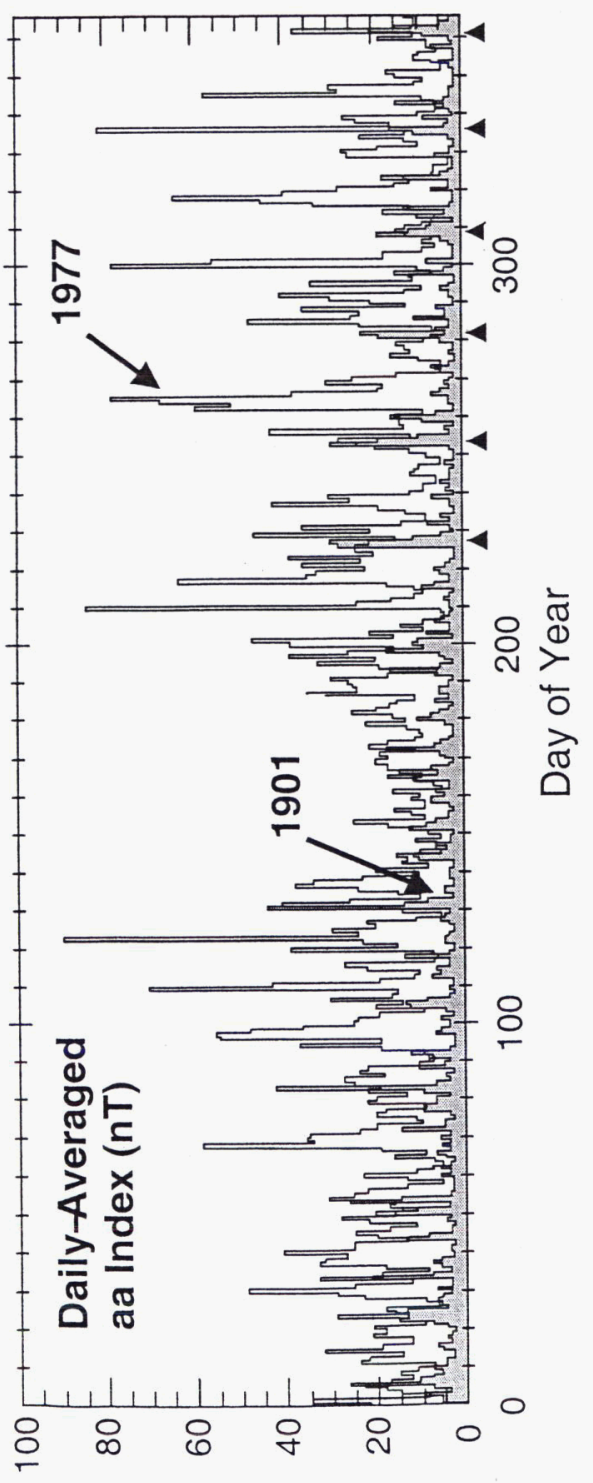




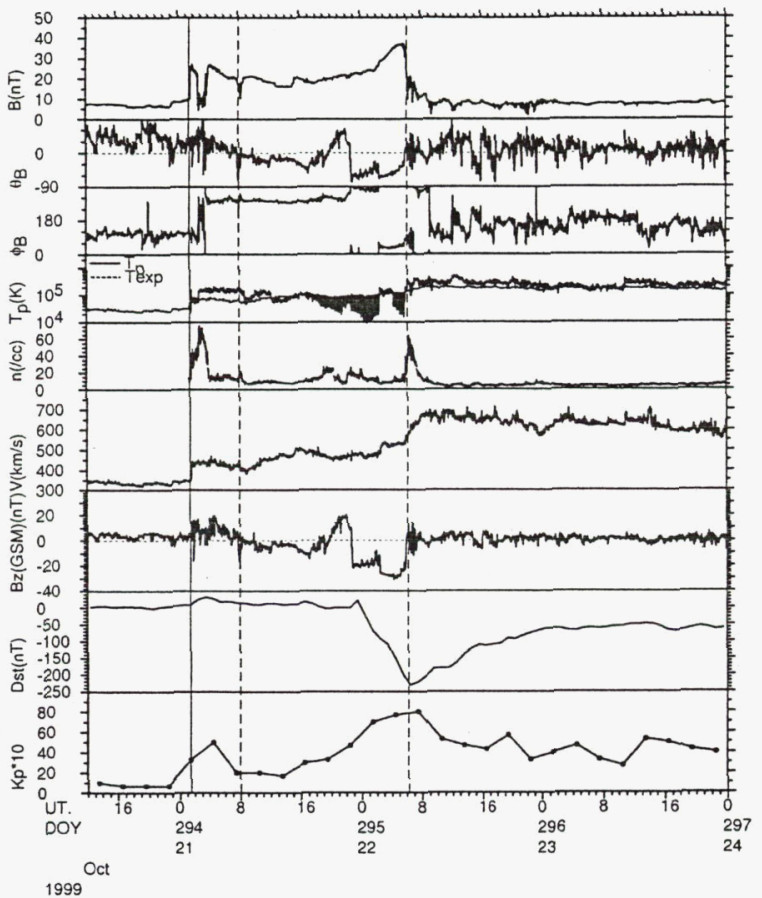

